\title{
Heartbeat: armed conflict and cardiovascular disease
}

Armed conflict affects a substantial number of people worldwide and often interferes with efforts to prevent and treat cardiovascular disease (CVD). Jawad and colleagues ${ }^{1}$ performed a systematic review that included 66 studies of adult civilian populations from 23 conflicts. Armed conflict was associated with increased blood pressure, lipid levels, and alcohol and tobacco use (figure 1). There also was weak evidence for an association with increased coronary, cerebrovascular and endocrine diseases. The authors conclude that post-conflict reconstruction should include attention to preventative measures to reduce the risk of CVD-related morbidity and mortality.

Ansbro and Perel $^{2}$ comment in an editorial that 'This review and a recent review of diabetes care in humanitarian crises both highlight the impact of the increasing global burden of non-communicable diseases (NCDs) on crisis settings and support advocacy work to bring NCDs, including CVD and the excess crisis-associated risks, squarely onto the agendas of humanitarian and development agencies, funders and the research community.' Main research priorities include rapid assessment tools, simple diagnostic tools and approaches, individual risk stratification, appropriate models of care, and ensuring availability and affordability of essential medicines and technologies.

Eating fish is recommended as part of a heart-healthy diet but there has been concern that the cardiovascular benefits of long-chain omega-3 polyunsaturated fatty acids (PUFA) might be offset by methylmercury levels in fish. In a study of 2199 men aged 42 to 60 years from the Kuopio Ischemic Heart Disease Risk Factor Study, Tajik and colleagues ${ }^{3}$ found that higher serum levels of long chain omega-3 PUFA were associated with a lower odds of exercise-induced myocardial ischaemia but only among men with a history of coronary heart disease (OR 0.10, 95\% CI 0.03 to 0.39 , p-trend $<0.001)$. In addition, higher hair mercury levels were associated with a higher odds of myocardial ischaemia (OR 1.62, 95\% CI 1.22 to 2.14 , p-trend $=0.002$ ) (figure 2).

Correspondence to Professor Catherine M Otto Division of Cardiology, University of Washington, Seattle, WA 98195, USA; cmotto@uw.edu

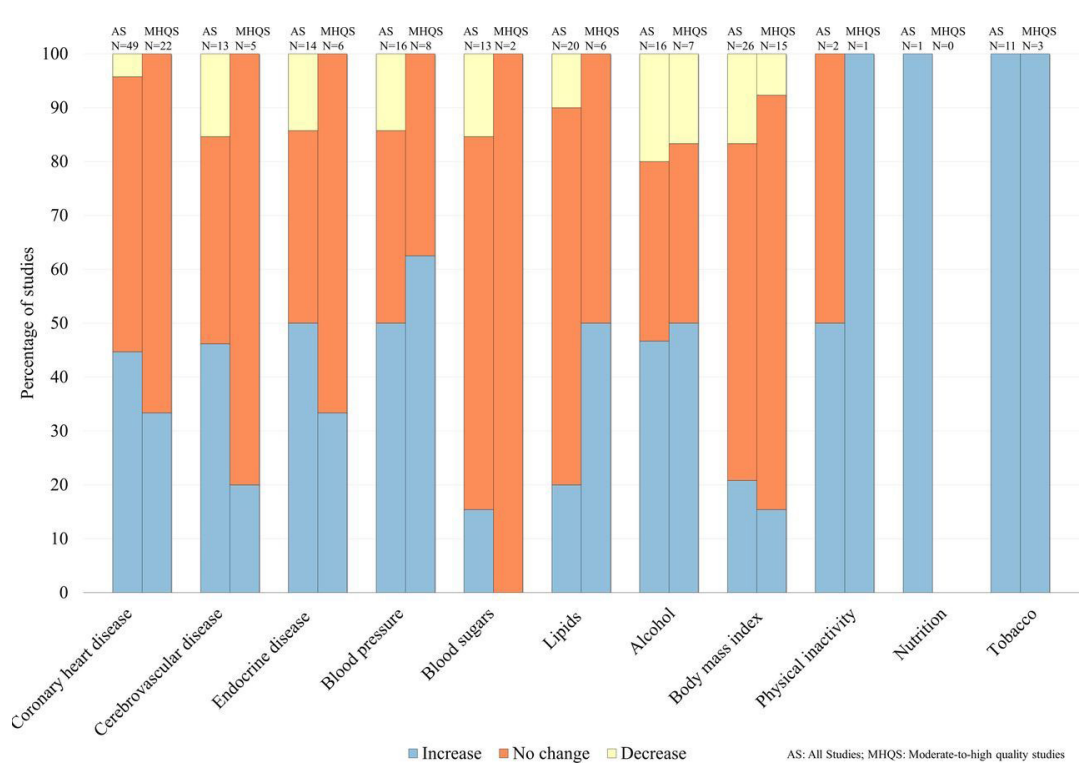

Figure 1 Exposure to armed conflict and non-communicable disease risk-summary of results by broader-level outcomes. Bars for all studies (AS) and moderate-to-high quality studies (MHQS); $\mathrm{n}=$ number of studies. blue for increase with armed conflict, orange for decrease and yellow for no change.

In an editorial, Pan and colleagues ${ }^{4}$ conclude that 'On balance, the current body of evidence suggests the benefits of moderate fish consumption on cardiovascular health very likely outweigh the risks of any potential harm attributable

to mercury exposure.' 'For those who are concerned about mercury exposure, risks can be further mitigated through consuming a diverse variety of fish, and avoidance of large predatory fish such as swordfish, shark and king mackerel. Of

\section{Subgroup}

$E P A+D P A+D H A$

All participants

Participants with history of CHD

Participants without history of CHD

EPA

All participants

Participants with history of CHD

Participants without history of CHD

DPA

All participants

Participants with history of CHD

Participants without history of CHD

DHA

All participants

Participants with history of CHD

Participants without history of CHD

MERCURY

All participants

Participants with history of CHD

Participants without history of CHD

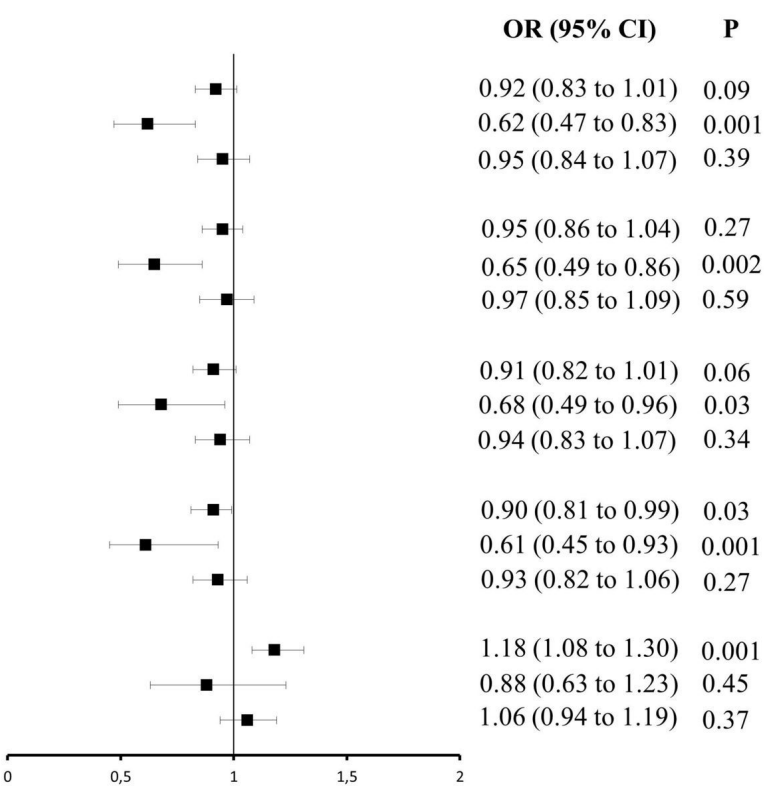

Figure 2 Multivariable adjusted odds of exercise-induced myocardial ischaemia in 1 SD change in the serum fatty acid and hair mercury concentrations (model 2). CHD, coronary heart disease; DHA, docosahexaenoic acid; DPA, docosapentaenoic acid; EPA, eicosapentaenoic acid. 

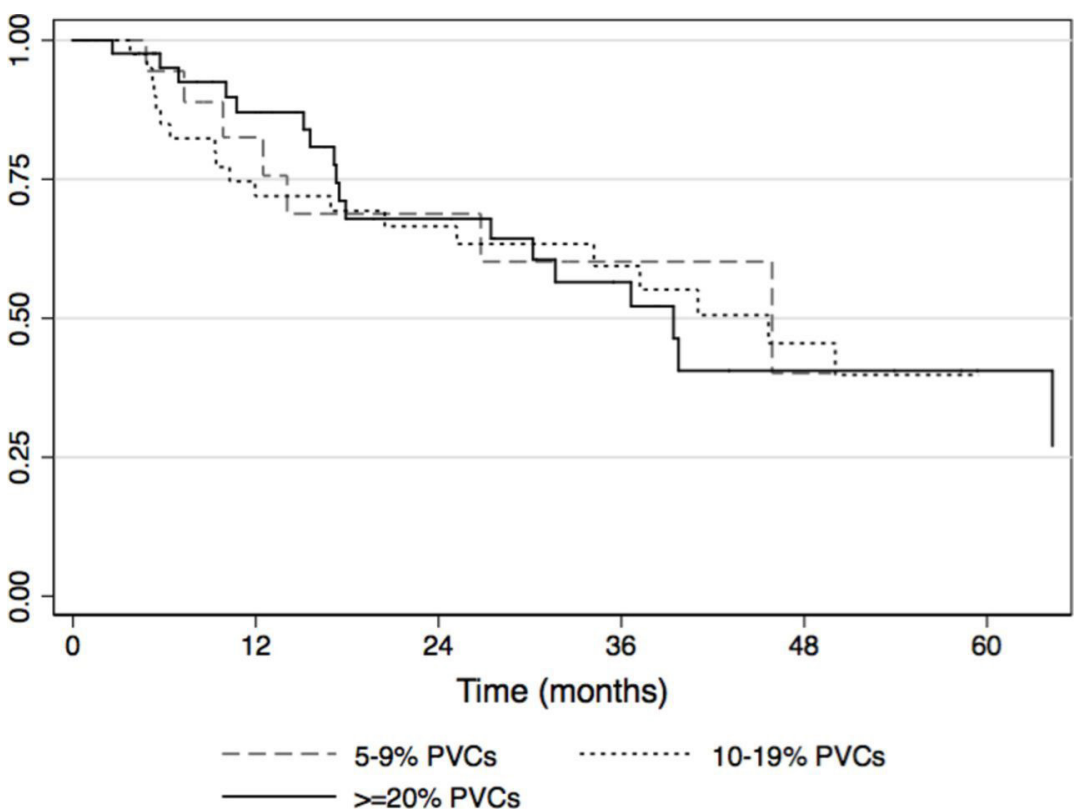

Figure 3 Time to reduction of PVCs to $<1 \%$, by initial PVC burden. PVC, premature ventricular complex.

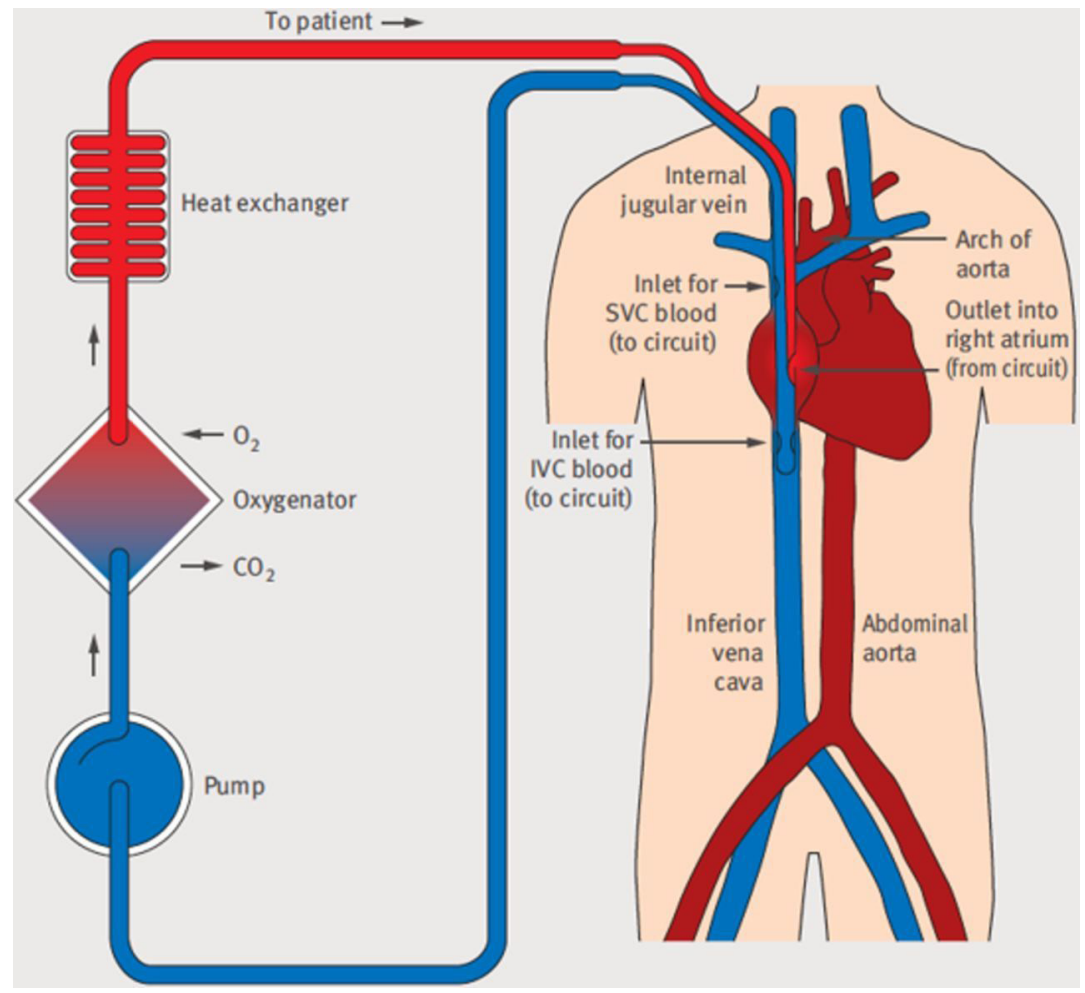

Figure 4 Dual lumen cannula version of veno-venous extracorporeal membrane oxygenation for respiratory failure: deoxygenated blood from both the superior and inferior venae cavae passes into one lumen of the double lumen cannula. blood flows in tubing to a pump and on to an oxygenator and heat exchange unit, before being returned to the right atrium through the second lumen with an oxyhaemoglobin concentration approaching $100 \%$. Here, oxygenated blood mixes with deoxygenated blood that has bypassed the double lumen cannula. This mixture of oxygenated and deoxygenated blood (oxyhaemoglobin saturation of around $80 \%$ ) is pumped by the heart through the non-functioning lungs into the aorta and on to the organs and tissues of the body. blue: intravascular deoxygenated blood; red: intravascular oxygenated blood; dark red: intravascular and intracardiac mixed oxygenated and deoxygenated blood; IVC, inferior vena cava; SVC, superior vena cava. Reproduced from Gaffney et al. ${ }^{9}$ note, although omega-3 PUFA supplements contain no mercury, they cannot replace the role of fish as part of a healthy diet.' They conclude that we work towards improved public health measures: 'Finally, since high mercury exposure can cause other health problems (eg, detrimental neurocognitive effects in infants), public policy should continue to reduce mercury contamination in the environment and fish, such as through reducing reliance and lowering pollution from coal power plants which is a major anthropogenic mercury source.'

Frequent premature ventricular complexes (PVCs) in patients with a normal left ventricular ejection fraction (LVEF) historically were considered to be benign. More recently, there has been concern that asymptomatic or minimally symptomatic frequent PVCs ( $\geq 5 \%$ in 24 hours) might be associated with adverse long-term consequences. In a single-centre cohort study of 100 consecutive patients with frequent PVCs, a normal LVEF and no evidence of myocardial disease on late-gadolinium enhanced cardiac magnetic resonance imaging (LGE CMR), Lee and colleagues ${ }^{5}$ found a spontaneous reduction to $<1 \%$ PVCs in $44 \%$ of patients at a median of 15 months follow-up, unrelated to PVC burden at presentation (figure 3). However, $4.3 \%$ of patients with a persistently elevated PVC burden developed an LVEF $<50 \%$ at an interval ranging from 53 to 71 months.

Roca-Luque and Mont ${ }^{6}$ summarise the evidence and conclude that 'the results of the study presented by Lee and colleagues ${ }^{5}$ suggest that a conservative 'wait and see' strategy of periodic LVEF evaluation in patients with frequent PVC, in the absence of structural heart disease (including LGE CMR in selected patients), can be reasonable due to the high likelihood of spontaneous resolution and the low rate of cardiac events and PVC-induced cardiomyopathy'.

The Education in Heart article in this issue $^{7}$ discusses the use of extracorporeal membrane oxygenation (ECMO) including which conditions can be managed with ECMO and the most common complications. This approach increasingly is used to stabilise critically ill patients with acute myocardial infarction, fulminant myocarditis, severe heart failure, intractable arrhythmias and for primary graft failure after heart transplantation (figure 4).

The Cardiology in Focus article in this issue $^{8}$ provides data on cardiology trainees participating in less than full time (LTFT) clinical training. Currently, about $15 \%$ of 
all UK physician trainees and about 4\% of UK cardiology trainees are LTFT, most often due to childcare responsibilities. LTFT cardiology trainees raise concerns about equitable training opportunities, limited opportunities in some subspecialties, expectations to work over the rostered hours, errors in pay and unfair allocation of on-call assignments. On the positive side, LTFT trainees report better work-life balance, excellent support from supervisors and training programme directors and the ability 'to continue a job they love, despite health or personal problems, when the alternative would have been a different career'.

Funding The authors have not declared a specific grant for this research from any funding agency in the public, commercial or not-for-profit sectors.
Competing interests None declared.

Patient consent for publication Not required.

Provenance and peer review Commissioned; internally peer reviewed.

(c) Author(s) (or their employer(s)) 2019. No commercial re-use. See rights and permissions. Published by BMJ.

\section{Check for updates}

To cite Otto CM. Heart 2019;105:1379-1381.

Heart 2019:105:1379-1381.

doi:10.1136/heartjnl-2019-315732

\section{REFERENCES}

1 Jawad M, Vamos EP, Najim M, et al. Impact of armed conflict on cardiovascular disease risk: a systematic review. Heart 2019;105:1388-94.

2 Ansbro É, Perel P. At the heart of the conflict. Heart 2019;105:1382-3.
3 Tajik B, Tuomainen T-P, Kurl S, et al. Serum long-chain omega-3 fatty acids, hair mercury and exerciseinduced myocardial ischaemia in men. Heart 2019;105:1395-401.

4 Pan X-F, Marklund M, Wu JH. Fish consumption for cardiovascular health: benefits from long-chain omega- 3 fatty acids versus potential harms due to mercury. Heart 2019;105:1384-5.

5 Lee AKG, Andrade J, Hawkins NM, et al. Outcomes of untreated frequent premature ventricular complexes with normal left ventricular function. Heart 2019:105:1408-13.

6 Roca-Luque I, Mont L. Frequent premature ventricular complexes and normal ejection fraction: to treat or not to treat? Heart 2019;105:1386-7.

7 Ali J, Vuylsteke A. Extracorporeal membrane oxygenation: indications, technique and contemporary outcomes. Heart 2019;105:1437-43.

8 Dobson R, Joshi A, Allen C, et al. Less than full-time training in cardiology. Heart 2019;105:1444-5.

9 Gaffney AM, Wildhirt SM, Griffin MJ, et al. Extracorporeal life support. BMJ 2010;341:c5317. 